\title{
APPLICATION OF WATER BEADS AS A NOVEL AND SIMPLE SORBENT FOR SMARTPHONE-BASED COLORIMETRIC DETERMINATION OF IRON IN WATER
}

\author{
Cristina B. Adamo ${ }^{\mathrm{a}}$, Ayandra S. Junger ${ }^{\mathrm{a}}$ and Dosil P. de Jesus ${ }^{\mathrm{a}, *,(1)}$ \\ aDepartamento de Química Analítica, Instituto de Química, Universidade Estadual de Campinas, 13083-970 Campinas - SP, Brasil
}

Recebido em 17/03/2021; aceito em 18/05/2021; publicado na web em 16/06/2021

\begin{abstract}
This work proposes a novel approach for colorimetric assays using ubiquitous and inexpensive water beads (WBs) made of a superabsorbent polymer (sodium polyacrylate). The high-water absorption capacity by the WBs was exploited for the preconcentration of iron ions from samples of tap, well, and lake waters. Moreover, the WBs also worked as a substrate for colorimetric detection of iron by the classical reaction of formation of Fe(II)-phenanthroline complex. Digital images acquired with a smartphone were used to obtain the color intensity of the WBs containing the orange color complex. The WBs were able to uptake and preconcentrate iron ions, allowing colorimetric detection with good linearity $\left(\mathrm{R}^{2}=0.9978\right)$ and limits of detection $(\mathrm{LOD})$ and quantification $(\mathrm{LOQ})$ of 0.02 and $0.07 \mathrm{mg} \mathrm{L}^{-1}$, respectively. The proposed method provided recoveries ranging from 93 to $111 \%$ for a sample of well water spiked with $0.15 \mathrm{mg} \mathrm{L}^{-1}$ of iron.
\end{abstract}

Keywords: colorimetric assay; digital image; sodium polyacrylate; superabsorbent polymer; water bead.

\section{INTRODUCTION}

Quick, easy, affordable, reliable, and real-time chemical analysis using portable analytical devices has gained attention in the last decades, particularly for clinical, environmental, food safety, and security applications. ${ }^{1-3}$ Among the detection techniques used in these devices, colorimetry has been proved to be suitable mainly because of its simplicity, requiring inexpensive instruments or even the human eyes to obtain colorimetric readouts. ${ }^{4-6}$ With the popularization of digital cameras and their integration into smartphones, colorimetric detection using digital images became attractive for quantitative analysis in portable analytical devices designed to fast and out-of-lab analysis. ${ }^{78}$ Generally, in these colorimetric assays, digital images of a colored solution or a substrate surface are acquired after a selective or specific colorimetric reaction involving the analyte. Paper substrate usually impregnated with a colorimetric reagent has been the most used for several analytical applications. ${ }^{9,10}$ However, this detection approach has also been used in textile-thread and polymer-based analytical devices. ${ }^{11,12}$ Although promising results were reported using the mentioned substrates, there is room for evaluating new materials that show physical-chemical properties to improve colorimetric detection performance. In this context, the superabsorbent polymers $(\mathrm{SAPs})^{13}$ and their remarkable water absorption capacity can be interesting to add a preconcentration step in colorimetric assays. SAPs are usually composed of polymer chains produced from acrylic monomers, generally polyacrylamide or sodium polyacrylate. Beads of SAP are commercially available as microspheres known as water beads (WB), which are employed in many household products, for decoration, like toys for kids, agriculture, and many other applications. ${ }^{13,14}$ Additionally, WBs are biodegradable, nontoxic, and can absorb and hold hundreds of times their weight in water. ${ }^{13}$ Nevertheless, this superabsorbent property of the WBs has been scarcely exploited in chemical analysis. Thus, few works are found in the literature reporting analytical applications of the WBs, as described by Xie et al., ${ }^{15}$ which synthesized poly(acrylamideco-itaconic acid) beads and applied this WB to preconcentrate and determine microorganisms in water samples.

\footnotetext{
*e-mail: dosil@unicamp.br
}

In this work, we described a novel and reliable colorimetric method using digital images and the superabsorbent properties of the WBs of sodium polyacrylate to preconcentrate iron ions from water samples. The same WB was also evaluated as an inexpensive and easy-to-use substrate to conduct the colorimetric reaction to form the complex Fe(II)-phenanthroline. The proposed method was evaluated for the determination of iron in samples of tap and natural water.

\section{EXPERIMENTAL PART}

\section{Reagents and materials}

All reagents were of analytical grade and used without further purification. Deionized water (DI, Milli-Q, Millipore) was used in all preparations of solutions and samples. Sulfuric acid, 1,10-phenanthroline hydrochloride ( $o$-phenanthroline), acetic acid, sodium acetate, ascorbic acid, and $\mathrm{FeNH}_{4}\left(\mathrm{SO}_{4}\right)_{2} \cdot 12 \mathrm{H}_{2} \mathrm{O}$ were purchased from Sigma Aldrich (St Louis, MO, USA).

Transparent WBs made of sodium polyacrylate with a spherical shape and a diameter of around $2 \mathrm{~mm}$ (dried) were commercially obtained in the local market of Campinas - Brazil.

\section{Standard and sample solutions}

A $50 \mathrm{mg} \mathrm{L}^{-1}$ of $\mathrm{Fe}(\mathrm{III})$ solution was prepared by dissolving an appropriate mass of $\mathrm{FeNH}_{4}\left(\mathrm{SO}_{4}\right)_{2} .12 \mathrm{H}_{2} \mathrm{O}$ in $0.1 \mathrm{~mol} \mathrm{~L}^{-1}$ sulfuric acid. By diluting this stock solution, iron standard solutions in concentrations varying from 0.05 to $0.50 \mathrm{mg} \mathrm{L}^{-1}$ were prepared in 10 $\mathrm{mL}$ volumetric flasks. Before being filled up with deionized water, the flasks were added $400 \mu \mathrm{L}$ of ascorbic acid solution $1 \%(\mathrm{~m} / \mathrm{v})$ to reduce the $\mathrm{Fe}$ (III) to $\mathrm{Fe}(\mathrm{II})$, and the $\mathrm{pH}$ was adjusted to 4.7 using $1 \mathrm{~mL}$ of acetate buffer solution $\left(0.5 \mathrm{~mol} \mathrm{~L}^{-1}\right)$. A blank solution was prepared using all the reagents except iron.

Samples of tap water, well water, and lake water were collected and filtered twice using membrane syringe filters $(0.22 \mu \mathrm{m}$ pore $)$. Before the colorimetric assay, a volume of $7.2 \mathrm{~mL}$ of the sample was added $0.8 \mathrm{~mL}$ of the ascorbic acid solution and $2 \mathrm{~mL}$ of the acetate buffer solution. 


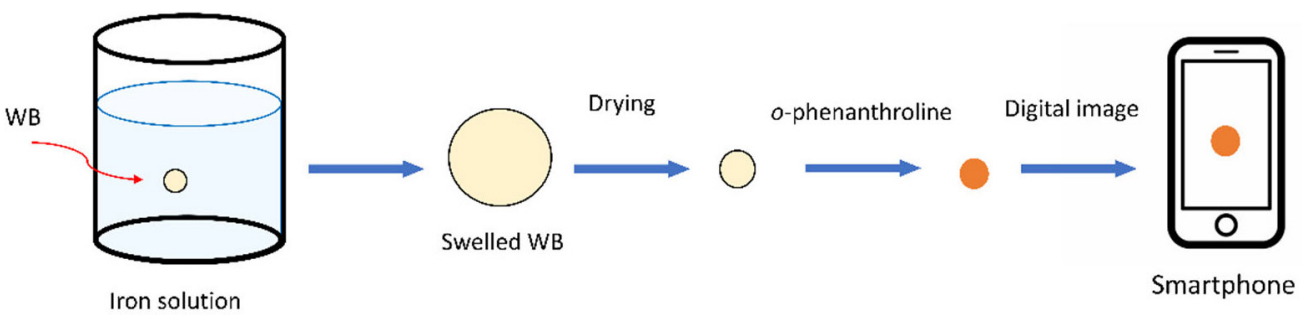

Figure 1. Schematic representation of the experimental procedure (not in scale)

Recovery tests were conducted using well water samples to evaluate the accuracy of the proposed method. For these tests, the water samples were spiked at a concentration of $0.15 \mathrm{mg} \mathrm{L}^{-1}$ using the standard iron solution. As described early, the spiked samples were added ascorbic acid and acetate buffer solution.

The samples, ascorbic acid, and standard iron solutions were prepared daily.

\section{Procedure for uptaking and colorimetric detection of iron}

Figure 1 shows a scheme of the experimental procedure for colorimetric detection of iron using WB. To $1 \mathrm{~mL}$ of the standard solutions or samples (prepared as described in the last section), one WB was added and remained for 30 min under mechanical stirring. Next, the sphere was removed from the solution, washed with DI water, and placed in an oven for $30 \mathrm{~min}$ at $90{ }^{\circ} \mathrm{C}$. In the following, a volume of $30 \mu \mathrm{L}$ of $0.25 \%(\mathrm{~m} / \mathrm{v}) o$-phenanthroline was pipetted over the WB surface. After $15 \mathrm{~min}$, the WBs were placed in the oven $\left(90^{\circ} \mathrm{C}\right)$ for $20 \mathrm{~min}$. In the sequence, the digital images were immediately obtained using a digital camera integrated into a smartphone model Samsung Galaxy A5 2017 (SM-A520).

The WBs were introduced (encrusted) into a white-color polymeric base (Styrofoam) that worked as a mechanical support for the WBs and improved the color contrast. This Styrofoam base was photographed without the flash function of the smartphone, and the focal distance was fixed to about $10 \mathrm{~cm}$. The obtained WB images were analyzed using the procedure described by Souza et al. ${ }^{12}$ with the software Corel PhotoPaint X8 (Corel Corporation, Canada). Briefly, the WB image obtained by the smartphone using the RGB system was converted to the CMYK system. After that, using the magenta color channel, the mean of the pixel intensity of the area corresponding to the WB in the image was obtained. This value could be associated with the iron concentration in the standard or sample solutions used in the colorimetric assay.

Absorption spectra of solutions of $\mathrm{Fe}(\mathrm{II})$-phenanthroline complex (0.05 to $0.5 \mathrm{mg} \mathrm{L}^{-1}$, in iron) were obtained using a UV-Vis spectrophotometer (Hewlett Packard, model HP89090A).

\section{RESULTS AND DISCUSSION}

\section{Optimization of the method}

During the iron uptake procedure, the WBs absorbed the solution and swelled, causing an increase in its diameter (from around 2 to $9 \mathrm{~mm}$ ), as depicted in Figure 2.

When the water in the swelled WBs was evaporated (heating), the spheres returned to their initial diameter, but the amount of iron (uptake) remained in the polymer. Considering that after the heating process, the WBs usually decreased their diameter from 9 to $2 \mathrm{~mm}$ and the volume of a sphere varied with the cube of its radius or diameter, the WB volume was decreased by around 91 -fold. We also found that each WB could absorb $339.2 \mathrm{mg}$ of water, corresponding to 30 -fold of its weight (about $11.9 \mathrm{mg}$ ). The WBs acquire an orange color after the reaction (Figure 1S, Supplementary Material section) between the iron

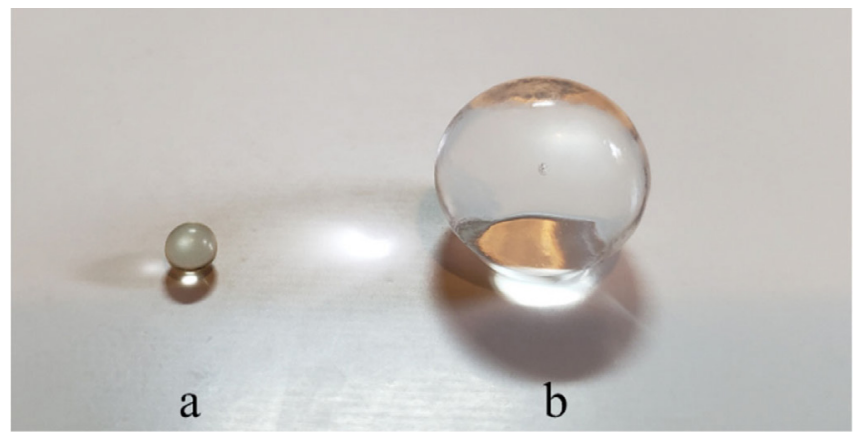

Figure 2. Images of WBs (a) before and (b) after swelling caused by water absorption

inside the polymer and the chromogenic reactant (o-phenanthroline). Figure 3 shows the WBs after the iron uptake procedures (using different iron concentrations) and the reaction of formation of the $\mathrm{Fe}(\mathrm{II})$-phenanthroline complex. As the color intensities of these WBs were obtained from a unique image, the illumination conditions were similar for all WBs encrusted on the Styrofoam base. This procedure simplified the image acquisition, but it cannot correct intra and interday changes in the light intensity of the environment. The used WBs had a low-intensity yellow/orange color that increased its intensity after the colorimetric assay using a blank solution. This increase in the background color most probably can be due to a slight degradation of the polymer because of the drying process using heating $\left(90^{\circ} \mathrm{C}\right)$. Nevertheless, the quantitative measurements were few affected by the developed background color of the WB. The heating of the WBs after the iron uptake and the addition of o-phenanthroline solution was necessary to reduce the time for the evaporation of the solutions that could require several hours to be complete at room temperature.

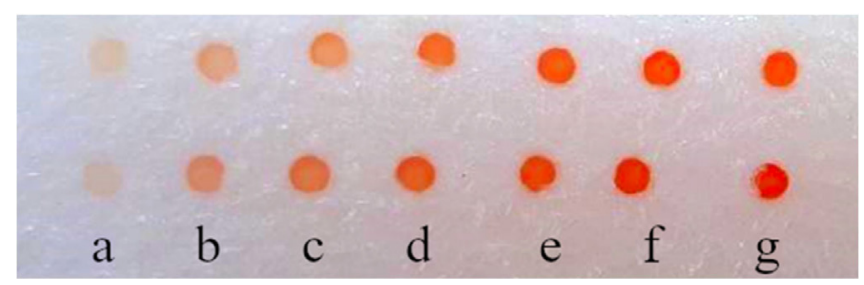

Figure 3. Image of the WBs obtained after the procedures of iron uptake (at different concentrations) and formation of the complex $\mathrm{Fe}(\mathrm{II})$-phenanthroline. Iron concentrations used in the uptake procedure: (a) $0 ;$; (b) 0.05 ; (c) $0.1 ;$ (d) 0.15; (e) 0.2; (f) 0.25; ( $g$ ) $0.3 \mathrm{mg} \mathrm{L} \mathrm{L}^{-1}$

The image analysis procedure was based on the work published by de Souza et al. ${ }^{12}$ that found that the CYMK color space provided better sensitivity when compared with the RBG system for detecting iron using the same reaction with o-phenanthroline. However, the color intensity was evaluated using the color channels cyan, yellow, and magenta in our work. For this study, colorimetric assays were carried out using iron solutions with concentrations of 0 (blank), 0.2 , and $0.5 \mathrm{mg} \mathrm{L}^{-1}$, and the images of the used WBs were analyzed using the different color channels, as depicted in Figure 2S. The color 
intensities using the cyan channel were similar for the different iron concentrations. On the other hand, the yellow and magenta channels allowed intensity variations with the iron concentrations. Although both color channels could be employed for the colorimetric detection, the magenta channel provided a smaller background intensity (blank solution) and a more linear response, so this channel was chosen for further experiments.

\section{Iron determination in water samples}

Once the colorimetric method was optimized, it was applied to determine the iron concentration in water samples. For comparison, absorption spectra of solutions of Fe(II)-phenanthroline complex ( 0.05 to $0.5 \mathrm{mg} \mathrm{L}^{-1}$, in iron) were obtained and shown in Figure $3 \mathrm{~S}$. The spectra revealed low intensity and a poor signal-to-noise ratio, even for the characteristic peak (at $510 \mathrm{~nm}$ ) for the iron complex. On the other hand, for iron solutions with concentrations ranging from 0.05 to $0.30 \mathrm{mg} \mathrm{L}^{-1}$, the proposed colorimetric method demonstrated better sensitivity and a linear response $\left(\mathrm{R}^{2}=0.9948\right)$, as depicted in the calibration curve showed in Figure 4. Moreover, the proposed method can be considered compatible with the green chemistry principles because it required low volumes of the sample $(1 \mathrm{~mL})$ and reagents (tens of microliters of o-phenanthroline solution). The high value of the intercept for the regression equation (Figure 4) was because of the background color (not subtracted) of the WBs, as early discussed.

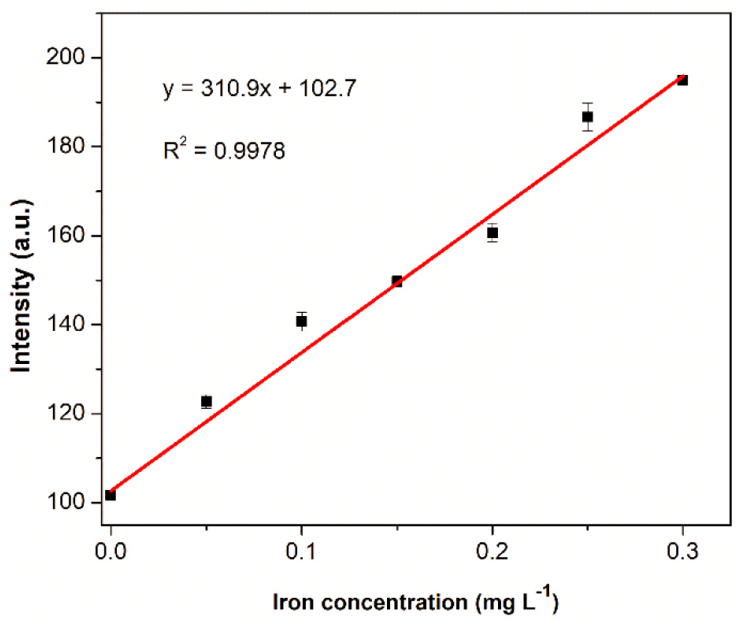

Figure 4. Calibration curve for the colorimetric detection of iron using WB. Error bars represent the standard deviation value for $(n=3)$ different images of the same WBs

The limits of detection (LOD) and quantification (LOQ) were respectively calculated as 3 and 10 standard deviations of the color intensity $(n=3)$ for colorimetric assays using a blank solution. Thus, the LOD was $0.02 \mathrm{mg} \mathrm{L}^{-1}$, and the LOQ was $0.07 \mathrm{mg} \mathrm{L}^{-1}$, comparable or better than those reported for other sensitive colorimetric methods for iron determination (Table 1S). ${ }^{12,16-21}$ Moreover, these limits can be considered suitable to detect iron concentrations in water above $0.30 \mathrm{mg} \mathrm{L}^{-1}$ that is the limit established by the Brazilian regulatory agency (CONAMA, Conselho Nacional do Meio Ambiente) ${ }^{22}$ and the American Public Health Association. ${ }^{23}$ The relative standard deviation for 12 colorimetric assays conducted on six different days was $14 \%$.

The iron concentrations in the analyzed water samples were found lower than the LOQ. However, the recovery test using the well water sample spiked with iron solution at a concentration of $0.15 \mathrm{mg} \mathrm{L}^{-1}$ resulted in recoveries $(n=7)$ ranging from 93 to $111 \%$, with an average of $104 \%$ and a standard deviation of $7 \%$. This accuracy can be considered interesting for quantitative analysis.

\section{CONCLUSIONS}

The WBs made of the superabsorbent sodium polyacrylate demonstrated to be an exciting absorbent for preconcentration of iron and a suitable substrate to conduct colorimetric assay using digital images. Moreover, these WBs are largely commercially available, inexpensive (about US\$ 3.5 for five thousand units), and non-toxic. The proposed colorimetric method using WBs showed good sensitivity, making it suitable for monitoring iron concentration in water samples. We believe this work can open many possibilities of applications of the WBs in chemistry, particularly for chemical analysis. For example, WBs could be chemically modified to improve the selectivity and the efficiency of extraction and preconcentration of ions or molecules before colorimetric or other detection methods.

\section{SUPPLEMENTARY MATERIAL}

Table 1S and the Figures 1S, 2S, and 3S can be freely accessed at http://quimicanova.sbq.org.br, in PDF format.

\section{ACKNOWLEDGMENTS}

The authors would like to acknowledge SAE-UNICAMP for the fellowships for A. S. Junger. This work was supported by the Fundação de Amparo à Pesquisa do Estado de São Paulo (FAFESP, grants n ${ }^{\circ}$ 2013/22127-2, 2014/50867-3, 2018/06478-3), the Conselho Nacional de Desenvolvimento Científico e Tecnológico (CNPq, grant $\mathrm{n}^{\mathrm{o}} 311430 / 2017-1$ and 305447/2019-0) and INCTBio (88887137482/2017-00). This study was financed in part (fellowship for C.B. Adamo) by the Coordenação de Aperfeiçoamento de Pessoal de Nível Superior-Brasil (CAPES)-Finance Code 001.

\section{REFERENCES}

1. Zhang, W.; Guo, S.; Pereira Carvalho, W. S.; Jiang, Y.; Serpe, M. J.; Anal. Methods 2016, 8, 7847.

2. Zarei, M.; TrAC - Trends Anal. Chem. 2017, 91, 26.

3. Li, Y.; Chen, Y.; Yu, H.; Tian, L.; Wang, Z.; TrAC - Trends Anal. Chem. 2018, 98, 190.

4. Liu, B.; Zhuang, J.; Wei, G.; Environ. Sci. Nano 2020, 7, 2195.

5. Gianini, G.; Mazzu-nascimento, T.; Stockton, A. M.; Carrilho, E.; Anal. Chim. Acta 2017, 970, 1.

6. Fernandes, G. M.; Silva, W. R.; Barreto, D. N.; Lamarca, R. S.; Lima Gomes, P. C. F.; Flávio da S Petruci, J.; Batista, A. D.; Anal. Chim. Acta 2020, $1135,187$.

7. Capitán-Vallvey, L. F.; López-Ruiz, N.; Martínez-Olmos, A.; Erenas, M. M.; Palma, A. J.; Anal. Chim. Acta 2015, 899, 23.

8. Grudpan, K.; Kolev, S. D.; Lapanantnopakhun, S.; McKelvie, I. D.; Wongwilai, W.; Talanta 2015, 136, 84.

9. Soda, Y.; Bakker, E.; ACS Sensors 2019, 4, 3093.

10. Sriram, G.; Bhat, M. P.; Patil, P.; Uthappa, U. T.; Jung, H. Y.; Altalhi, T.; Kumeria, T.; Aminabhavi, T. M.; Pai, R. K.; Madhuprasad; Kurkuri, M. D.; TrAC-Trends Anal. Chem. 2017, 93, 212.

11. Weng, X.; Kang, Y.; Guo, Q.; Peng, B.; Jiang, H.; Biosens. Bioelectron. 2019, 132, 171 .

12. de Souza, F. R.; Duarte Junior, G. F.; de Tarso, P.; Coltro, W. K. T.; Quim. Nova 2014, 37, 1171.

13. Zohuriaan-Mehr, M. J.; Kabiri, K.; Iran. Polym. J. (English Ed. 2008, $17,451$.

14. Khan, S. B.; Ali, F.; Asiri, A. M.; Int. J. Hydrogen Energy 2019, 45, 1532.

15. Xie, X.; Bahnemann, J.; Wang, S.; Yang, Y.; Hoffmann, M. R.; Sci. Rep. 2016, 6, 1. 
16. Nie, B.; Zhao, S.; Deng, M.; Yu, P.; Yang, Y.; Lei, W.; Yin, L.; J. Braz. Chem. Soc. 2021, 32, 599.

17. Choodum, A.; Sriprom, W.; Wongniramaikul, W.; Spectrochim. Acta, Part A 2019, 208, 40.

18. Peng, B.; Chen, G.; Li, K.; Zhou, M.; Zhang, J.; Zhao, S.; Food Chem. 2017, 230, 667.

19. Ballesteros, J. I.; Caleja-Ballesteros, H. J. R.; Villena, M. C.; Microchem. J. 2021, 160, 105652.
20. Barros, J. A. V. A.; Oliveira, F. M. de; Santos, G. de O.; Wisniewski, C.; Luccas, P. O.; Anal. Lett. 2017, 50, 414.

21. Ninwong, B.; Dungchai, W.; Asian J. Chem. 2014, 26, 133.

22. http://www2.mma.gov.br/port/conama/legiabre.cfm?codlegi $=459$, accessed at May 2021.

23. Standard Methods for the Examination of Water and Wastewater; American Public Health Association, American Water Works Association, Water Environment Federation, 1999. 